\title{
Estado del conocimiento y principales amenazas de los humedales boscosos de agua dulce de Chile
}

\author{
Knowledge status and principal threats to freshwater forested wetlands of Chile
}

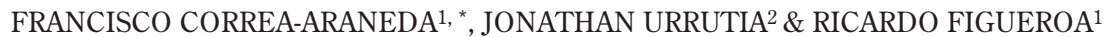 \\ ${ }^{1}$ Laboratorio de Bioindicadores de Calidad de Agua, Unidad de Sistemas Acuáticos, Centro de Ciencias Ambientales \\ EULA-Chile, Universidad de Concepción, Casilla 160-C, Concepción, Chile \\ 2 Departamento de Botánica, Facultad de Ciencias Naturales y Oceanográficas, Universidad de Concepción, \\ Casilla 160-C, Concepción, Chile \\ *Autor correspondiente: franciscocorrea@udec.cl
}

\begin{abstract}
RESUMEN
Los humedales boscosos de agua dulce son áreas naturalmente inundadas o saturadas, que sustentan vegetación boscosa hidrófila, están presentes en todo el mundo, y en Chile son conocidos como "pitrantos, hualves o hualhues". El presente estudio entrega el estado del conocimiento de estos humedales en Chile, mediante una descripción de sus características biológicas, fisicoquímicas e hidrodinámicas, identificando las principales amenazas para su conservación y las principales necesidades de investigación. Estos ambientes pueden presentar inundación permanente o temporal, dependiendo de un conjunto de procesos microclimáticos, biológicos y edafológicos. Su vegetación, del tipo hidrófila boscosa, es dominada por especies de la familia Myrtaceae. Tanto el comportamiento hídrico como la estructura vegetacional destacan dentro de los aspectos que determinan directamente las características fisicoquímicas del agua y los patrones de distribución de las comunidades biológicas. Los humedales boscosos de Chile han sido ampliamente estudiados desde un punto de vista vegetacional y florístico, pero carecen de estudios limnológicos, hidrológicos o faunísticos básicos, de lo cual deriva un total desconocimiento en relación a su funcionamiento a nivel ecosistémico y los efectos que pueden tener las actividades humanas en sus cuencas (e.g., agrícola, ganadera, forestal) sobre sus componentes hídricos y biológicos. A pesar de ser ecosistemas de gran relevancia cultural y ecológica, se identifican importantes falencias en cuanto a su estado de conservación, al no encontrarse protegidos por las actuales herramientas de conservación existentes en el país. Por lo tanto, emergen como ecosistemas únicos, de relevancia mundial, prácticamente desconocidos en Chile y de gran interés para el desarrollo de numerosas líneas de investigación, aunque su alta susceptibilidad ante perturbaciones de origen antrópico amenaza su permanencia en el tiempo.
\end{abstract}

Palabras clave: agua dulce, Chile, cuencas, humedal boscoso, vegetación hidrófila.

\begin{abstract}
Forested freshwater wetlands are naturally flooded or saturated areas with hydrophilic forest vegetation which is worldwide distributed and is known as "pitrantos, hualves o hualhues" in Chile. This paper gives to know the state of art of these wetlands in Chile, through a description of its biological, physical-chemical and hydro-dynamic characteristics, identifying the main threats to its conservation and the main research needs. These environments can be permanently or temporarily flooded, depending on microclimatic, biological and edaphical processes. Its vegetation is dominated by species of the family Myrtaceae. Both hydric behavior and vegetation structure are major aspects that directly determine the water physical-chemical characteristics and the distribution patterns of biological communities. Chile's forested wetlands have been studied broadly from a vegetation and floristic point of view, but basic studies on limnology, hydrology or fauna are lacking, which results on a total lack of knowledge about its functioning at the ecosystem level and the effects that human activities on basins (e.g., agricultural, stockbreeding, forest) could have on its hydric and biological components. In spite of being ecosystems of great cultural and ecological significance, important weakness can be identified on its state of conservation, as they are not protected by conservation tools that exist in Chile nowadays. Therefore, forested wetlands emerge as unique ecosystems of global importance, nearly unknown in Chile and of great interest on developing an important number of research lines, even though their high susceptibility facing anthropic disturbances threaten their continuance.
\end{abstract}

Key words: basins, Chile, forested wetland, fresh water, hydrophyte vegetation. 


\section{INTRODUCCIÓN}

Para el concepto de humedal se han elaborado numerosas definiciones, aunque se ha llegado al consenso de que este tipo de ecosistema corresponde a la zona de transición entre ambientes húmedos $\mathrm{y}$ ambientes generalmente secos, presentando características de ambos, por lo cual no pueden ser clasificados categóricamente como acuáticos ni terrestres (Barbier et al. 1997). Una de las definiciones más utilizadas es la elaborada por la Convención Relativa a los Humedales de Importancia Internacional (Ramsar 1996), que fue formulada con el objetivo central de conservar y usar de manera racional los recursos hídricos y la fauna que sustenta. Dicha definición indica que estos ambientes corresponden a extensiones de marismas, pantanos, turberas o aguas de régimen natural o artificial, permanentes o temporales, estancadas o corrientes, dulces, salobres o saladas, incluyendo las extensiones de agua marina cuya profundidad en marea baja no exceda de seis metros. De lo que deriva el amplio espectro de ecosistemas acuáticos que pueden enmarcarse dentro de ella. Sin embargo, en términos prácticos (e.g., investigación), es necesario acotarla, basándose en algunos aspectos de su funcionalidad, morfometría e hidrología, con el fin de diferenciarlos de ambientes lacustres, fluviales o marinos, los cuales solo son comparables entre sí por el hecho de ser ecosistemas acuáticos, pero muy distintos en su ecofisiología. Definiciones que intentan aproximarse a este concepto coinciden en que corresponden a ecosistemas de aguas someras $(<2 \mathrm{~m})$ o con suelos saturados, los cuales presentan un régimen hídrico permanente o temporal, con una baja tasa de recambio y abundante materia orgánica vegetal que se descompone lentamente, que permite la existencia y desarrollo de biota acuática característica (Cowardin et al. 1979, Boavida 1999, Davis et al. 2006, Mitsch \& Gosselink 2000, 2007).

Dicha definición reduce considerablemente el número de ecosistemas que pueden ser incluidos dentro del concepto de humedal, pero la gran diversidad de este tipo de ambientes sigue generando la necesidad de categorizarlos, sobre todo al momento de realizar estudios más específicos. Investigadores de numerosos países, como Inglaterra, Canadá, Sudáfrica, Estados Unidos y Chile, han realizado esfuerzos para clasificar estos ecosistemas, sobre las características físicas, químicas, biológicas e hidrológicas (e.g., Cowardin et al. 1979, Brinson 1993, Warner \& Rubec 1997, Dini et al. 1998, Hauer \& Smith 1998, Ramírez et al. 2002, Clausen et al. 2006). No obstante, sigue siendo escaso el conocimiento sobre la funcionalidad de estos sistemas, lo que emerge como una tarea pendiente que debe ser prontamente abordada, con la finalidad de aportar a su adecuada gestión y conservación.

Los humedales entregan múltiples servicios ecosistémicos a la sociedad, destacando, entre otros, la conservación de la biodiversidad acuática, regulación microclimática, abastecimiento de agua potable, diversidad paisajista y embellecimiento escénico, amortiguación hidráulica de inundaciones invernales y tsunamis, oferta de espacios para actividades recreativas y educación ambiental, transporte, recursos energéticos e inclusive tratamiento de aguas residuales (Ewel 1997, Bolund \& Hunhammar 1999, Ramsar 2006, Mitsch \& Gosselink 2007), cumpliendo por lo tanto una función relevante en las estrategias de desarrollo socioeconómico sostenible. Por otra parte, a pesar de constituir solo una pequeña fracción del total de la superficie terrestre, estos ambientes poseen una gran diversidad biótica y de biomasa por unidad de área, en comparación con otros ecosistemas acuáticos (e.g., lagos, lagunas, ríos) y terrestres (Naiman \& Décamps 1997), siendo considerados los ecosistemas de mayor riqueza biológica en la tierra (Innis et al. 2000), y recientemente han sido denominados como ambientes de gran complejidad ecológica e importantes para su conservación (Davis et al. 2006, Mitch et al. 2009).

La vegetación hidrófila es común a todos los humedales, sin embargo, hay un tipo de humedal incluido en la mayoría de las clasificaciones elaboradas a nivel mundial, que se distingue por el tipo de vegetación hidrófila predominante, denominados humedales boscosos, los cuales han sido definidos como áreas naturalmente inundadas o saturadas, que soportan un importante componente de vegetación de tipo boscosa, adaptada a un suelo generalmente saturado y/o pobremente aireado (Lugo 1990). 
Aunque a nivel mundial, estudios recientes sobre los humedales boscosos han mostrado interés por aspectos sobre la flora (e.g., Amigo et al. 2004), vegetación (e.g., RodríguezGonzález et al. 2004) o su funcionamiento (Sampaio et al. 2008); en Chile aún existe un bajo interés sobre este tipo de ecosistemas, y desconocimiento sobre las principales temáticas en las que han centrado sus esfuerzos los especialistas, no siendo fácil definir políticas nacionales de conservación (e.g., CONAMA 2005), al enmarcarse en modelos internaciones de los cuales se desconoce su aplicabilidad en la realidad diversa y singular de Chile.

Es debido a lo anterior, que el objetivo de la presente revisión es presentar el estado del conocimiento de los humedales boscosos de agua dulce de Chile, basados en información respecto a sus características biológicas, fisicoquímicas e hidrodinámica, e identificar las principales amenazas para su conservación, que determinen las principales necesidades de investigación.

\section{HUMEDALES BOSCOSOS}

Los humedales boscosos se encuentran representados a nivel mundial por los humedales boscosos del ciprés de los pantanos (Taxodium distichum (L.) L. Rich), ubicados principalmente en Estados Unidos, y los manglares costeros de Centroamérica, el Caribe y Asia, que reciben su nombre por estar constituidos por especies arbóreas llamadas comúnmente mangles (Rhizophora spp.; Li \& Lee 1997, Hauenstein et al. 1999, Berlanga-Robles \& Ruiz-Luna 2004). De estos últimos, se tiene un amplio conocimiento y son considerados como los humedales boscosos más representativos a nivel mundial. Han sido estudiados desde muchos puntos de vista, incluyendo descripciones florísticas, faunísticas y limnológicas, caracterizaciones de sus condiciones edáficas, hídricas, fisicoquímicas y biogeográficas e incluso sobre su valor socioeconómico, productivo, ambiental y estado de conservación (e.g., Thong \& Sasekumar 1984, Altenburg \& Van Spanje 1989, Chong et al. 1990, Alongi \& Christoffersen 1992, Duke 1992, Aikanathan \& Sasekumar 1994, Feller \& Mathis 1997, Ashton 2002, Feller 2002, Díaz et al. 2004, Faunce \& Serafy 2006).
En Chile, los humedales boscosos de agua dulce son menos conocidos y están representados por los "pitrantos", también conocidos como "hualves" o "hualhues", que en lengua mapuche significa ciénaga o pantano (Ferriere 1982). Se distribuyen entre Coquimbo y Puerto Montt $\left(29^{\circ} 54^{\prime}-41^{\circ} 38^{\prime}\right.$ S) a través de la depresión central, cordillera de la Costa, a mediana altura en la cordillera de los Andes (Ramírez 1982, San Martín et al. 1988) y en la isla de Chiloé $\left(41^{\circ} 00^{\prime}-42^{\circ} 30^{\prime}\right.$ S; Ramírez \& San Martín 2005).

Estos humedales se sitúan principalmente en fosas tectónicas, fondos de quebradas $\mathrm{u}$ hondonadas con suelos que presentan mal drenaje (Varela 1981, Ramírez et al. 1983, Castro 1987, San Martín et al. 1988, Solervicens \& Elgueta 1994). Estas condiciones geomorfológicas y edáficas son las que posibilitan el aporte de aguas subterráneas (Villa-Martínez \& Villagrán 1997), presentando un régimen hídrico de anegamiento temporal. Aunque también, en los casos en que se encuentran asociados a esteros o ríos (Hauenstein et al. 2002), este régimen generalmente es de tipo permanente, y el nivel del agua en ambas situaciones puede presentar fluctuaciones intra e interanuales (FernándezAlaéz et al. 2004).

\section{Régimen hídrico, procesos biológicos y variables fisicoquimicas}

El régimen hídrico o hidroperíodo es uno de los componentes más importantes en el funcionamiento de estos ecosistemas. Es un descriptor de los humedales, en base a las fluctuaciones de la profundidad, duración y frecuencia, en un periodo de un año (Keddy 2000). Las variaciones en el nivel del agua, tanto en humedales permanentes como temporales, teóricamente son el resultado de la interacción de un conjunto de complejos procesos hidrológicos, geológicos y biológicos, tales como precipitación, infiltración, evaporación y transpiración vegetal (Fig. 1; Fisher et al. 2000, Arle 2002, Mitsh \& Gosselink 2007, Tavernini 2008), y de las recargas y flujos de aguas subterráneas y superficiales (Siegel 1988, Roulet 1991, Devitoa et al. 1996).

Respecto a los humedales boscosos de anegamiento temporal, se ha documentado que este periodo puede durar entre cuatro 
y ocho meses (Ramírez et al. 1995), ligado estrechamente a la época invernal, aunque dicha duración puede ser mayor o menor, en relación directa con las condiciones climáticas, edáficas y geomorfológicas de la zona donde se ubiquen. Durante el periodo invernal, las precipitaciones permiten el ascenso de la napa freática y el escurrimiento de las aguas desde las laderas adyacentes hacia las zonas más bajas, en donde se ubican estos ecosistemas, alcanzando profundidades de hasta $2 \mathrm{~m}$. En cambio en la época estival, cuando el espejo de agua desaparece por efecto de la evapotranspiración e infiltración, sus suelos pueden presentar entre un $50 \mathrm{y}$ $60 \%$ de contenido de humedad, con una napa freática muy cercana a la superficie $(20-30 \mathrm{~cm}$; Ramírez et al. 1983, Del Valle-Arango 2003). En cambio, en terrenos más bajos o de menor permeabilidad, el agua puede permanecer todo el año, variando en profundidad superficial de acuerdo a cambios climáticos o relaciones hidrodinámicas mencionadas, definiendo de esta forma los humedales permanentes.

Todos los procesos anteriores constituyen unos de los factores limitantes de la actividad biológica, controlando, entre otros aspectos, la productividad primaria, la colonización y la formación de estructuras comunitarias presentes en estos ambientes (Ramírez et al. 1983, Tavernini et al. 2005). De esta forma distintas comunidades de vertebrados, invertebrados y plantas están asociadas de manera alternada a la fase seca y a la húmeda (Angeler et al. 2010). La alternación de estas fases, que a menudo ocurre de manera irregular, crea condiciones de vida extremas, que favorecen la presencia de una biodiversidad única, altamente especializada, y en su mayoría restringida exclusivamente a este tipo de hábitats (Holland \& Jain 1981, Zedler 1987, Boix et al. 2001, Hillmann \& Quinn 2002, Arle 2002, Coops et al. 2003, Nicolet et al. 2004, Fraga i Arguimbau 2008), y que para Chile es prácticamente desconocida.

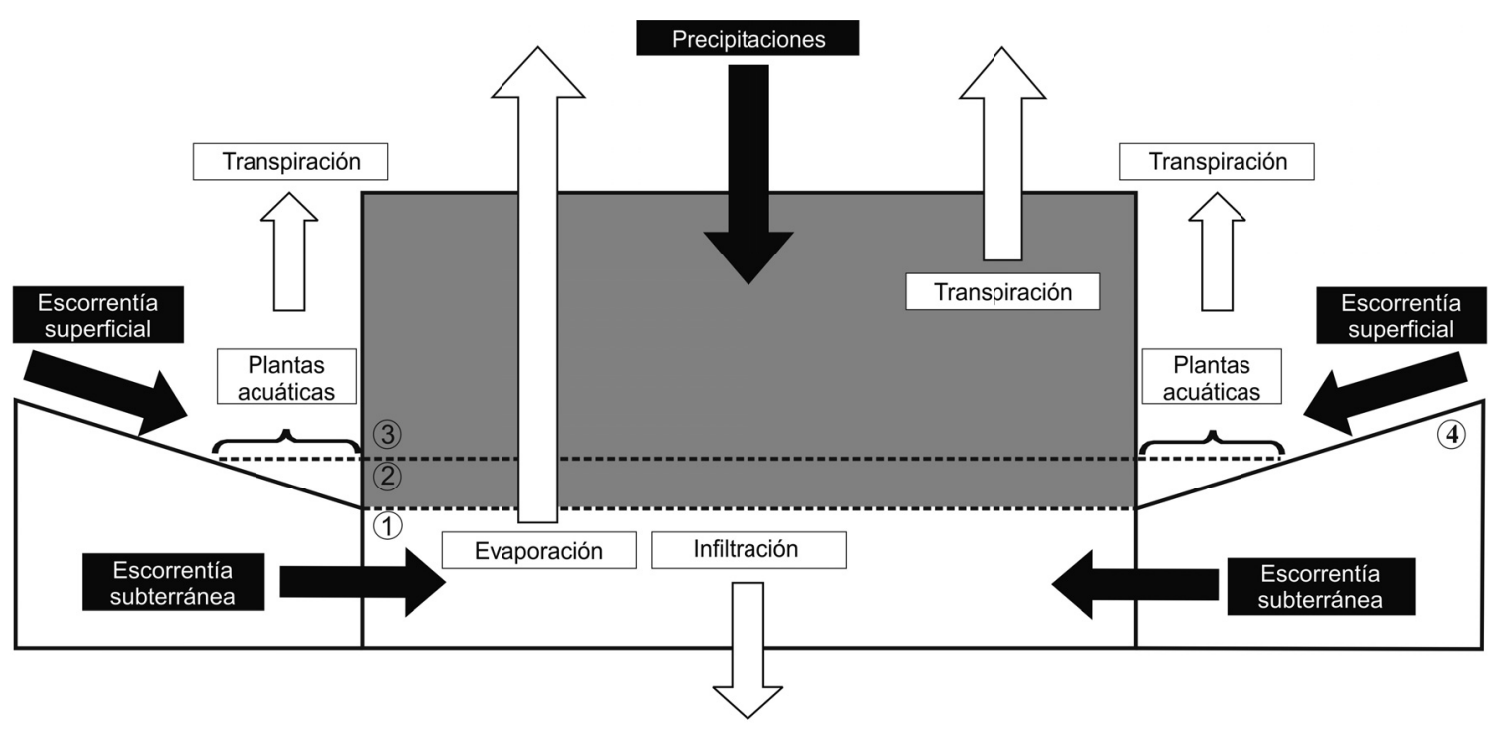

Fig. 1: Modelo conceptual que representa los procesos (precipitación, escorrentía superficial, escorrentía subsuperficial, infiltración, evaporación y transpiración) que influyen en la dinámica hídrica de los humedales boscosos. Las flechas indican los flujos hídricos de entrada (flechas negras) y salida (flechas blancas); $1=\mathrm{Nivel} \mathrm{de}$ agua durante época estival; 2 = Nivel de agua durante época invernal; 3 = Zona ocupada por vegetación boscosa; 4 = Ladera adyacente.

Conceptual model that represents the processes (precipitation, surface runoff, subsurface runoff, infiltration, evaporation and transpiration) influencing the water dynamics of forested wetlands. Arrows indicate water flows of input (black arrows) and output (white arrows); 1 = Water level during summer season; $2=$ Water level during winter season; $3=$ Zone occupied by forested vegetation; 4 = Adjacent hillside. 
En términos de la funcionalidad de estos sistemas, el hidroperíodo es uno de los factores relevantes que sinergia procesos como la descomposición de la materia orgánica (Lugo et al. 1990), ya que condiciones inundadas propician condiciones aeróbicas y un suelo saturado que favorecen una descomposición a una tasa más elevada que en aquellas condiciones anaeróbicas en un sustrato seco (Lockaby \& Wallbridge 1998, Cronk \& Fennessy 2001), e influye además en la calidad del alimento proveniente del detritus y en la liberación de nutrientes que contribuye en la mantención de un tipo de ecosistema altamente productivo (Wiggins et al. 1980). Al respecto, la comunidad bacteriana sería entonces la principal encargada del reciclaje de los nutrientes de estos ambientes, sin embargo, en Chile aún son inexistentes los estudios respecto a la dinámica de los descomponedores en relación al procesamiento de la materia orgánica de estos sistemas acuáticos altamente dependientes del bosque que sustenta. El régimen hídrico determina además muchas de las variables fisicoquímicas del agua, determinantes en los patrones de distribución de las comunidades biológicas, entre las cuales destacan particularmente el pH y la conductividad (Nicolet et al. 2004), esta última presenta un aumento antes y después de la recarga de los humedales temporales, lo cual no es solo resultado del cambio en la concentración por efecto del decrecimiento del volumen de agua, sino que además de la redilución de las sales minerales presentes en el sustrato que son resuspendidas después de periodos de precipitaciones o mediante su escorrentía desde las laderas adyacentes (Arle 2002, Tavernini et al. 2005, Tavernini 2008). Sin embargo, este proceso puede manifestarse de una manera menos acentuada en estos ambientes, por efecto de la protección generada por el estrato arbóreo dominante, la cual intercepta y disminuye la velocidad de caída de las gotas, permitiendo su escurrimiento a través de las distintas estructuras vegetales (Huber \& Oyarzún 1990, Huber \& López 1993, Echeverría et al. 2007). Por otra parte, Comin \& Williams (1994) sugieren que los patrones de circulación del agua, dinámica de nutrientes y las interacciones agua-sedimento son esencialmente similares entre ambientes permanentes y temporales, sin embargo, en estos últimos, debido a la inestabilidad generada por el secado y llenado de los humedales, estas se encuentran más estrechamente relacionadas con las condiciones ambientales (e.g., clima, actividad antrópica), y por lo tanto, su susceptibilidad ante perturbaciones de tipo antrópico es mayor.

\section{HUMEDALES BOSCOSOS DE CHILE}

\section{Estructura y composición boscosa}

Dentro de las características relevantes de estos ecosistemas, destaca la presencia de un dosel arbóreo cerrado de entre 18 y $20 \mathrm{~m}$ de altura (Ramírez et al. 1995), compuesto por especies hidrófilas siempreverdes, dentro de las cuales se encuentran representantes de la familia de las mirtáceas, de los géneros Myrceugenia, Blepharocalyx, Luma y Tepualia. Además, es común la presencia del canelo Drimys winteri J.R. et G. Forster (San Martín et al. 1988, Hauenstein et al. 2002), que al igual que los géneros anteriormente mencionados, requiere de un ambiente con altos niveles de humedad.

La vegetación de estos ambientes es considerada del tipo azonal (San Martín et al. 1988), ya que contrasta con las típicas formaciones boscosas influenciadas por factores climáticos de la zona donde se ubican. Por el contrario, las especies de mirtáceas que dominan estos ecosistemas, están principalmente determinadas por factores hidrológicos y edáficos (e.g., hidroperíodo, tipo de sustrato), los cuales además influyen en los patrones de dominancia de las especies, que permite reconocer a la fecha 6 asociaciones florísticas características de estos ambientes: (1) La asociación Blepharocalyo-Myrcengenietum exsuccae (Ramírez et al. 1995), del humedal boscoso de temu-pitra, distribuido desde Victoria hasta Puerto Montt (Oberdorfer 1960, Villagrán 1982, Ramírez et al. 1983), (2) la asociación Lumo-Myrceugenietum exsuccae (Pisano 1954), del humedal de canelo-pitrachequén, ubicado en la depresión central de la región de Valparaíso (Pisano 1954, Villagrán 1982), (3) la asociación Lumetum (Ramírez 1982) del humedal de arrayán (Luma apiculata (DC.) Burret), que prospera en la cordillera de los Andes de la Región de Los Lagos (Ramírez 1982), (4) la asociación Perseo-Myrceugenietum 
exsuccae (San Martín et al. 1990), del humedal de canelo-lingue-pitra, ubicada en zonas húmedas de la cordillera de la Costa en la región de los bosques maulinos (Ramírez et al. 1995), (5) la asociación Tepualietum stipulariae (Hueck 1978), del humedal de tepú, presente en la isla de Chiloé, con avanzadas septentrionales en la cordillera Pelada, al sur del río Valdivia y al norte del cerro Oncol (Lépez 1998), y (6) la más recientemente descrita asociación Caldcluvio-Lumetum gayanae del humedal de chinchin-canelo, ubicado en la cuenca inferior del río Valdivia (San Martín et al. 2006).

La distribución, estructura y composición del componente boscoso de estos ecosistemas determinan las dinámicas físicas, químicas y biológicas de los humedales, tanto a escala temporal como espacial (Fennessy et al. 2001, Reiss 2006). Es así como el porcentaje de cobertura vegetal de los humedales boscosos, como ocurre en la mayoría de los ecosistemas acuáticos, está inversamente relacionado con la temperatura (Hauer \& Lamberti 2006). Bosques bien conservados disminuyen la cantidad de radiación solar que llega de manera directa hacia el espejo de agua, reduce el impacto del viento, por lo que la temperatura media del aire en el interior del bosque durante el día es inferior a los espacios adyacentes, creando un microclima que amortigua las oscilaciones diurnas de temperatura y reducen la temperatura del medio acuático. La presencia de agua y la evapotranspiración de las plantas contribuyen también a una elevada humedad relativa (CHS 2008). Esto es particularmente relevante, ya que este parámetro corresponde a un factor que controla la mayoría de los procesos vitales para los organismos, afecta las propiedades químicas y físicas de otros factores abióticos en un ecosistema y es determinante en la pérdida de agua mediante el proceso de evaporación (Kadlec 2006, Betancout et al. 2009). Cambios en la temperatura del agua, ocasionado por una modificación de la estructura vegetacional, provocarían además una paulatina disminución de la profundidad, afectando procesos esenciales para el funcionamiento de estos ecosistemas, mediante un aumento de la actividad microbiana y respiratoria, y por lo tanto de la tasa de descomposición de la materia orgánica. De igual forma, la modificación en la dinámica de dichos procesos generaría una rápida disminución en la cantidad de oxígeno disuelto en el agua (Reddy \& DeLaune 2008), consecuentemente se pierde la capacidad de sumidero de carbono que poseen los humedales, alterando los ciclos naturales frente a un escenario poco alentador de cambio climático (Boyero et al. 2011).

Debido al bajo porcentaje de luz que penetra hacia el estrato inferior, este tipo de vegetación minimiza la presencia de sotobosque (San Martín et al. 1988, 2006), y se espera que otros tipos de comunidades vegetales (e.g., fitoplancton), también se encuentren pobremente representados. Por lo tanto, el estrato boscoso sería el componente encargado de la producción primaria, que mediante el detritus foliar constituye el mayor aporte energético a estos ecosistemas. Además provee hábitat, alimento, sitios de anidación y refugio para un importante número de especies de fauna silvestre (e.g., insectos, aves, anfibios, reptiles y mamíferos; Baran \& Hambrey 1998, Ashton, 2002, Cronk \& Fennessy 2001, Nagelkerken et al. 2008). Entre los grupos faunísticos, y en relación a su distribución y requerimientos, destacan algunas especies con graves problemas de conservación, como la guiiña (Leopardus guigna Molina 1782) y el huillín (Lontra provocax Thomas 1908), cuyas categorías corresponden a Vulnerable y En Peligro respectivamente (IUCN 2010). Por otra parte, la porción sumergida del bosque permite la formación de islotes a través del espejo de agua (Duberstein \& Conner 2009), los que entregan una gran diversidad de microhábitats para la fauna acuática (e.g., macroinvertebrados bentónicos), configurando por lo tanto un ambiente altamente heterogéneo. Al respecto, estudios experimentales (Vivian-Smith 1997) y de campo (Koponen et al. 2004) indican que lo anterior implica una gran riqueza de especies raras, en comparación con otros tipos de humedales. Sin embargo, aún no se ha comprobado si dicha relación es igualmente aplicable para los humedales de Chile.

\section{Estado del arte}

Hasta la fecha se han publicado 15 estudios realizados en humedales boscosos de Chile (Tabla 1), los que incluyen un total aproximado de 68 unidades de humedales, distribuidos entre las regiones de Coquimbo y de Los Ríos 
(Fig. 2). Ellos han sido generalmente tratados en la literatura como bosques pantanosos, y por consiguiente el interés se ha centrado en conocer las características del dosel arbóreo y los componentes asociados a él. $\mathrm{Al}$ respecto, las primeras descripciones de estos ambientes fueron realizadas por Reiche (1907) y Berninger (1929), más tarde Pisano (1954) los incluyó en la asociación vegetal Myrceugenietum, seguida de la más completa revisión de estos ambientes, la cual fue realizada por Oberdorfer (1960), quien además creó el sintaxón Temo-Myrceugenietum exsuccae. Recién a finales de la pasada década MuñozPedreros \& Möller (1997) los incluyeron, de acuerdo a la clasificación de la Convención de Ramsar, en la categoría Xf: "humedales boscosos de agua dulce”, aunque sin realizar estudios al respecto.

Estudios más específicos fueron realizados principalmente desde un punto de vista vegetacional y/o florístico (e.g., Ferriere 1982, San Martín et al. 1988, 1990, 1992a, 1992b, Ramírez et al. 1983, 1995, Villa-Martínez \& Villagrán 1997, González et al. 2003), con un total de siete estudios en la zona costera de las regiones del Libertador Bernardo O’Higgins y del Maule (Fig. 2). Según Ramírez et al. (1995), los humedales boscosos del centro-sur de Chile poseen un total de 250 especies de plantas vasculares, dos musgos y una hepática, cuya distribución taxonómica se reparte en tres Briophyta, 20 Pteridophyta, una Pinophyta, 180 Magnoliopsida y 49 Liliopsida (Fig. 3B). Cabe señalar también que de este universo la mayor parte son elementos nativos con 193 especies $(76 \%)$ y en menor medida aparecen los introducidos con 60 especies (24\%) (Fig. 3A). Este último número, sin embargo, es bastante alto y denota una fuerte intervención antrópica al interior de estos ecosistemas, especialmente cuando este estudio tiene más de una década, asociado a un fuerte desarrollo forestal del país. Además, es importante indicar que en los principales trabajos que han tenido como objetivo la clasificación y ordenación de la vegetación a nivel nacional, los humedales boscosos han sido tratados someramente. Donoso (1981) no hace alusión a ellos en la descripción del tipo forestal siempreverde,

TABLA 1

Listado de estudios realizados en humedales boscosos de Chile, agrupados por zona y temática.

List of studies conducted in the forested wetlands of Chile, grouped by zone and thematic.

\begin{tabular}{lll}
\hline Zona & \multicolumn{1}{c}{ Temática } & \multicolumn{1}{c}{ Referencia } \\
\hline Centro & Entomología & Solervicens (1973) \\
& Fitosociología & Jerez et al. (1977) \\
& Flora y fitosociología & San Martín et al. (1988, 1992b) \\
& Historia vegetacional & Villa-Martínez \& Villagrán (1997) \\
Centro-Sur & Flora & Reiche (1907) \\
& & Berninger (1929) \\
& Fitosociología & Pisano (1954) \\
& & Oberdorfer (1960) \\
& Entomología & Solervicens \& Elgueta (1994) \\
& Revisión & González et al. (2003) \\
Sur & Fitosociología & Ramírez et al. (1983) \\
& & San Martín et al. (2006) \\
& Flora & Ramírez et al. (1995)
\end{tabular}


en donde podría haberlos definido como un subtipo. Del mismo modo, Luebert \& Pliscoff (2006), en su sinopsis vegetacional de Chile, no los consideran dentro de ninguna de sus clasificaciones, y solo Gajardo (1995) se refiere a ellos, quien describe una asociación formada por el canelo y el temu, como parte de la región del bosque caducifolio de la frontera.

Desde un punto de vista faunístico se han realizado solo tres estudios entomológicos, específicamente sobre insectos de follaje (e.g., Solervicens 1973, Jerez et al. 1977, Solervicens \& Elgueta 1994), los cuales incluyeron 32 humedales boscosos, en un gradiente longitudinal desde Illapel ( $31^{\circ} 34^{\prime}$ $70^{\circ} 52^{\prime}$ S) hasta Valdivia (39 $43^{\prime}-72^{\circ} 52^{\prime}$ S) (Fig. 2). Lo anterior es particularmente relevante,

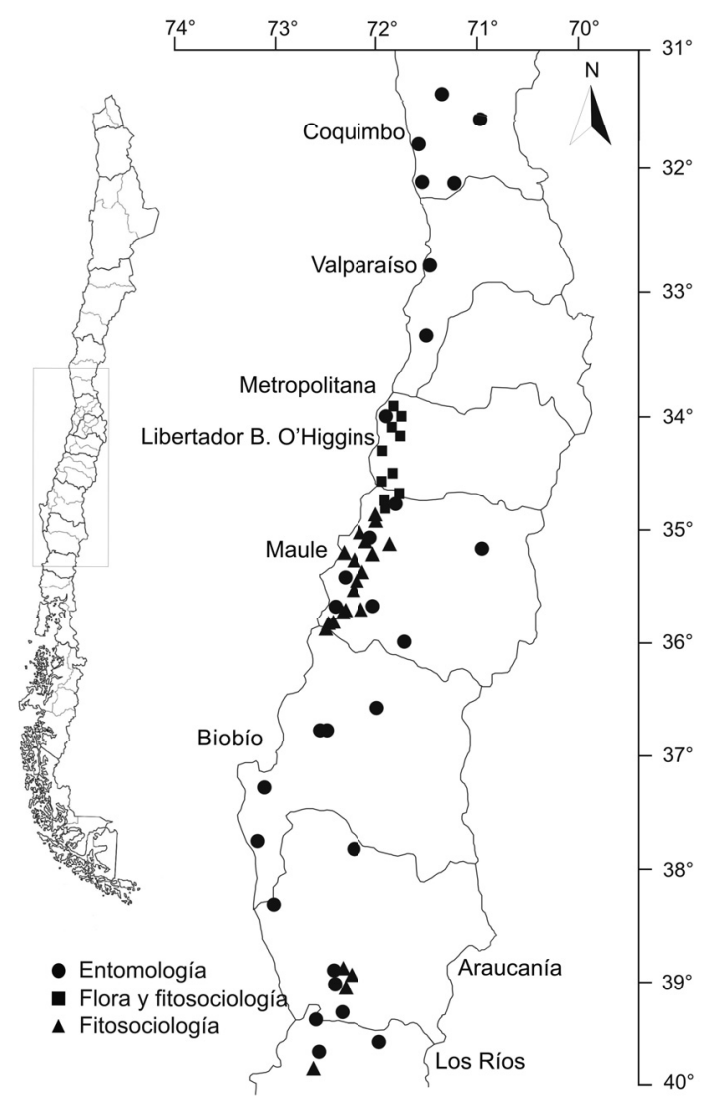

Fig. 2: Mapa de la distribución espacial de los principales humedales boscosos de agua dulce estudiados en Chile.

Map of the spatial distribution of the major freshwater forested wetlands studied in Chile. ya que dicho estudio abarcó la mayoría de los tipos de humedales boscosos que han sido descritos hasta la fecha, y que fueron clasificados en base a la dominancia de especies vegetales, tal como se menciona en párrafos anteriores de la presente revisión. Dichos estudios permitieron identificar un total de 654 taxa, distribuidos en dos agrupaciones de insectos (agrupación Norte Chico-V Región y agrupación Sur) de acuerdo a la variación climática a través del eje longitudinal del país, en las cuales dominaban hemípteros y coleópteros, cuyos representantes presentaron dependencia con ciertas plantas típicas de la comunidad boscosa (e.g., mirtáceas), y posiblemente pueden ser exclusivos de estos ambientes. Sumado a lo anterior, los autores concluyen que la homogeneidad vegetacional y microclimática de los humedales boscosos, crean condiciones óptimas para el desarrollo tanto de la entomofauna, como de otros grupos faunísticos, actuando como concentradores y reservorios de gran parte de la biota local (Solervicens \& Elgueta 1994).

\section{Principales amenazas}

A pesar de la importancia que se le asigna a estos ecosistemas, por su gran biodiversidad, la mayor parte de ellos está sometido a numerosos tipos de impactos, siendo considerados como uno de los ecosistemas más susceptibles ante perturbaciones a escala de cuenca (Peña-Cortés et al. 2006). Dentro de las principales causas de intervención, destaca la obtención de suelos aptos para la agricultura, existiendo, incluso, programas regionales con subsidio estatal para cumplir dicho objetivo (Ramírez et al. 1983, Solervicens \& Elgueta 1994, Ramírez et al. 1995, Ojeda 1998, Hauenstein et al. 1999), o para el establecimiento de proyectos inmobiliarios. En ambos casos los humedales son drenados y/o rellenados debido al proceso de expansión poblacional, transformándolos en canales o pozas temporales receptoras de basura (Figueroa et al. 2009). Además son utilizados como fuentes de extracción de leña para uso doméstico (temu y pitra), lo que genera la destrucción del hábitat mediante la apertura del dosel, implicando una mayor penetración de la luz solar, y por consiguiente cambios en las características químicas del agua. 
Los espacios creados facilitan la invasión espontanea de especies exóticas, generando una disminución en la diversidad de hábitats y modificando la estructura y composición arbórea (Davis \& Froend 1999). Si bien este tipo de perturbaciones han sido responsables de forma directa de la degradación o pérdida total de gran parte de los humedales, un grupo de ellos han permanecido en buen estado de conservación al encontrarse en lugares de difícil acceso (e.g., fondo de quebradas) (Solervicens \& Elgueta 1994). Otro tipo de perturbaciones que afectan de manera indirecta a estos ambientes es el cambio de uso de los suelos adyacentes para la agricultura, silvicultura o ganadería, cuyos procesos generan contaminación difusa por el uso de pesticidas, fertilizantes y la generación de compuestos nitrogenados de la ganadería. Estos elementos llegan a los cuerpos de agua de manera intermitente, mediante los procesos de arrastre superficial o lixiviación, en función de la estacionalidad de las actividades de aplicación o fenómenos de precipitaciones (Alfaro \& Salazar 2005, Chalar 2006).

El aporte de nutrientes, tales como el fósforo y el nitrógeno, hacia cuerpos de aguas superficiales, acelera el proceso de eutroficación de estos ecosistemas (Tundisi \& Matsumura-Tundisi 1990). En el caso de los humedales boscosos, conlleva a su paulatina degradación mediante una disminución en la transparencia de la columna de agua (Lampert \& Sommer 1997), en los niveles de oxígeno disuelto (Carpenter et al. 1998) y por la rápida proliferación de algas tóxicas (blooms) (Bobbink et al. 2006), especialmente en las zonas del borde, donde el espejo de agua se encuentra mayormente expuesto debido a la menor densidad del componente arbóreo, la cual es provocada en la mayoría de los casos por la intervención del dosel con fines extractivos.

De igual forma, la eutroficación de los humedales tiene severos efectos negativos, que son de gran relevancia para el ser humano, principalmente mediante la pérdida de variados servicios ecosistémicos, dentro de los cuales se incluyen el uso para consumo humano, regadío, variadas aplicaciones industriales o para recreación (Carpenter et al. 1999). Dicha situación es aún más extrema cuando el origen de estos efectos es debido al reemplazo del bosque nativo por monocultivos de especies exóticas (Bratton 1990, Serrano \& Serrano 1995, Muñoz-Pedreros \& Möller 1997), pues al estar relacionados de manera inversa con la cantidad, distribución y disponibilidad de los recursos hídricos (Echeverría et al. 2007), debido al mayor requerimiento que tienen las especies exóticas por dicho recurso gracias a su rápido crecimiento y metabolismo, implican una merma en los aportes de agua hacia estos ecosistemas. Lo anterior, en conjunto con un escenario de cambio climático global (e.g., variaciones de la temperatura) y las características someras de estos ambientes, pueden además provocar la modificación del régimen hídrico, reduciendo la frecuencia, duración y tasa de inundación o desecación de estos ecosistemas (Ramírez et al. 1995, Gafny \& Gasith 1999, Sala et al. 2000, Brönmark \& Hansson 2002, Perroti et al. 2005, Angeler \& Moreno 2007, Hulsmans et al. 2008, Brooks 2009).
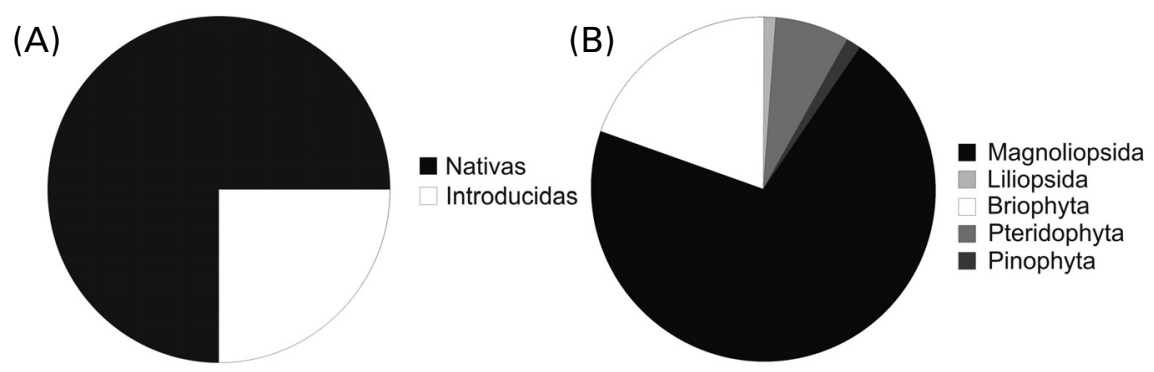

Fig. 3: Porcentaje de especies nativas e introducidas (A) y formas de vida (B) de las plantas descritas para los humedales boscosos de Chile.

Percentage of native and introduced species (A) and lifestyles (B) of the plants described for forested wetlands of Chile. 
Aunque los efectos que las actividades humanas pueden tener sobre los cuerpos de agua dulce en general son numerosos y han sido bien descritos en la literatura. En Chile, los estudios enfocados en caracterizar o evaluar dichos impactos sobre los humedales son escasos, recientes, principalmente de la última década, y se han enfocado en diversos tipos de humedales (e.g., Hauenstein et al. 1999, Muñoz-Pedreros 1993, 2004, Peña-Cortés et al. 2006, Zegers et al. 2006, Figueroa et al. 2009), excepto los humedales boscosos. Y en ningún caso los esfuerzos se han centrado en determinar las relaciones de causas y efectos de dichas actividades sobre los procesos y funciones específicas, así como tampoco en cuantificar la pérdida de estos sistemas, principalmente porque no existe un catastro claro y la mayor parte de ellos aún no han sido estudiados desde ningún punto de vista.

\section{Estado de conservación}

Respecto a la conservación de estos ecosistemas, Chile cuenta con un Sistema Nacional de Área Silvestres Protegidas del Estado (SNASPE), que representa la principal herramienta de protección de la biodiversidad, mediante la administración y manejo de áreas naturales distribuidas a través de todo el territorio nacional. Si bien cuenta con 34 Parques Nacionales, 49 Reservas Nacionales y 16 Monumentos Naturales (CONAF 2010) que actualmente administra la Corporación Nacional Forestal de Chile, solo en el Parque Nacional Vicente Pérez Rosales (Región de Los Lagos) se encuentra protegida una pequeña fracción de humedales boscosos correspondiente a la asociación temu-pitra (Serey \& Villaseñor 1977).

Chile además se encuentra suscrito al Convenio de Ramsar, el cual tiene por objetivo proteger y conservar a nivel mundial los humedales a través de su uso racional, mediante la implementación tanto de medidas locales como de cooperación internacional. A nivel mundial existen 1953 sitios Ramsar, equivalentes a 190455433 millones de hectáreas (Ramsar 2011), aproximadamente el $33.4 \%$ de la superficie total de humedales existentes. Al menos el $53 \%$ de estos sitios posee una porción de humedal boscoso dentro de sus límites. En Chile existe un total de 12 de estos sitios (358989 hectáreas), los cuales se distribuyen a través de todo el territorio (Ramsar 2011). No obstante, ninguno de ellos corresponde a un humedal del tipo boscoso, o presenta al menos una porción de estos dentro de sus límites.

\section{Importancia cultural}

Estos humedales forman parte importante de las tradiciones de los pueblos originarios, y producto de su cosmovisión son considerados espacios sagrados y respetados a lo largo de Chile (Grunewald et al. 2009), sobre todo en la Región de La Araucanía y la frontera con la Región del Biobío, donde el pueblo mapuche le otorga la presencia de energías o fuerzas naturales y espirituales (newen y ñeh), reciben el nombre de "menoko" y son fuente importante de plantas medicinales (Durán et al. 1997). Además son considerados como lugares de nacimiento de agua (vertientes), a los cuales solo es posible ingresar previa autorización de un ngen-ko (dueño del agua; Sánchez 2003). Al respecto, estos humedales han asegurado el suministro de agua para la población rural mediante la protección de las napas freáticas (Urrutia 2008), ya que dicho recurso comúnmente es obtenido a través de pozos. Lo anterior es aún más relevante para la Región de La Araucanía, ya que es la segunda región con más población rural (32\%) a nivel nacional (INE 2002).

Otro aspecto de arraigo corresponde a la vegetación que compone los humedales boscosos (mirtáceas), que dan origen a algunos de los nombres de las ciudades de la Región de La Araucanía a partir de su significado en la lengua mapudungun, en zonas en que estos ambientes probablemente cubrían grandes extensiones del territorio. Dentro de las cuales encontramos a las comunas de Temuco y Lumaco, cuyos nombres provienen de designaciones del mapudungun, lengua originaria del pueblo mapuche, y ambas presentan la terminación "ko", que significa agua o estero. Temuco es la actual capital de la Región de La Araucanía, y cuyo significado proviene del mapudungun "temúko", compuesta por "temu", la mirtácea arbórea de madera muy dura (Blepharocalyx cruckshanksii), que en conjunto con la terminación "ko" significa estero o zona 
cubierta por agua donde hay árboles de temu. De igual forma ocurrió con la comuna de Lumaco, cuya toponimia proviene del mapudungun "lumáko", compuesta por "luma”, el árbol mirtáceo de madera muy dura (Amomyrtus luma), y que junto a la terminación "ko", se refiere al estero o zona cubierta por agua donde hay luma (San Martín 1997).

Este conjunto de características relevantes, sumadas a los aspectos físicos y biológicos descritos en secciones anteriores, los han llevado a ser considerados en la Estrategia Regional de Conservación y Uso Sustentable de la Biodiversidad (CONAMA 2002), como lugares de alta importancia natural y cultural.

\section{Especialistas}

En primer lugar destaca el grupo liderado por el Dr. Carlos Ramírez, del Instituto de Botánica de la Universidad Austral de Chile, y el que encabeza el Dr. José San Martín del Departamento de Ciencias Biológicas de la Universidad de Talca, quienes han sido los principales exponentes sobre aspectos fitosociológicos y vegetacionales de estos ambientes. Por otra parte, se encuentra un grupo de expertos dirigido por el Dr. Jaime Solervicens del Instituto de Entomología de la Universidad Metropolitana de Ciencias de la Educación, quienes profundizaron en el conocimiento sobre la entomofauna terrestre de estos ambientes, dejando abierta la posibilidad de generar nuevos estudios respecto al mismo grupo taxonómico, pero en su ciclo de vida acuático o mediante estudios comparativos respecto a la comunidad de insectos en ambos estados de su desarrollo. Si bien dichos autores entregaron importantes aportes al conocimiento biológico de estos humedales, sus contribuciones fueron aisladas en el tiempo, y no se registran aportes recientes.

\section{CONCLUSIONES}

Los humedales boscosos de agua dulce corresponden a ecosistemas únicos, de relevancia mundial y prácticamente desconocidos en Chile, siendo de esta forma un sustrato único para desarrollar numerosas líneas de investigación. A pesar de lo anterior, grupos científicos ligados al estudio de los humedales, no han derivado esfuerzos en estudiar de manera más profunda dicho tipo de ecosistema. Motivo por el cual los mayores avances en su investigación los han logrado un grupo de expertos enfocados únicamente a dos líneas de investigación, de acuerdo a los grupos taxonómicos en los cuales son especialistas (plantas e insectos).

Por ello, destacan grandes vacíos de información respecto a su funcionamiento, principalmente aspectos sobre su hidrología, patrones de distribución de las comunidades acuáticas, condiciones fisicoquímicas del agua y cuantificación de áreas remanentes. Además, son inexistentes todo tipo de estudios faunísticos mayores (e.g., aves, mamíferos, reptiles, anfibios, peces), así como una caracterización y cuantificación de los efectos de las perturbaciones de origen antrópico sobre el estado natural de estos ambientes, por lo que constituyen los ecosistemas de agua dulce menos estudiados en Chile.

Los esfuerzos de conservación a través del SNASPE debieran al menos considerar la protección de la mayor variedad posible de ecosistemas. Esto hace referencia directa a la diversidad a nivel paisajístico, lo cual asegure las condiciones para una conservación de la diversidad ecosistémica que ofrecen los humedales boscosos que desaparecen día a día. Las herramientas con que cuenta nuestro país en términos de protección del patrimonio biológico, deben ser instrumentos eficientes al resguardo de nuestra singular biodiversidad y que la generación de conocimiento a través de la investigación en esta y otras disciplinas sirva para cumplir dicho objetivo, sin embargo, la pérdida de estos ambientes se mueve a una velocidad mayor y la ausencia de estudios sugiere que deben ser prontamente abordados a través de programas de investigación específicas. Su susceptibilidad ante las perturbaciones antrópicas indican que posiblemente puedan desaparecer antes que puedan ser caracterizados o simplemente identificados como una ecotipo acuático único.

AGRADECIMIENTOS: Estudio financiado por el proyecto CONAF 035-2010, del Fondo de Investigación del Bosque Nativo de la Corporación Nacional Forestal. Agradecimientos especiales a Rodrigo Correa, Andrés Moraga, Reinaldo Rivera y Miguel Gatica por sus importantes correcciones del manuscrito y aportes en la revisión bibliográfica. 


\section{LITERATURA CITADA}

AIKANATHAN S \& A SASEKUMAR (1994) The community structure of macroalgae in a low shore mangrove forest in Selangor, Malaysia. Hydrobiologia 285: 131-137.

ALFARO M \& F SALAZAR (2005) Ganadería y contaminación difusa, implicancias para el sur de Chile. Agricultura Técnica 65: 330-340.

ALONGI DM \& P CHRISTOFFERSEN (1992) Benthic infauna and organism-sediment relations in a shallow, tropical coastal area: influence of outwelled mangrove detritus and physical disturbance. Marine Ecology Progress Series 81: 229-245.

ALTENBURG W \& T VAN SPANJE (1989) Utilization of mangroves by birds in Guinea-Bissau. Ardea 77: 57-74.

ANGELER DG \& JM MORENO (2007) Zooplankton community resilience after presstype anthropogenic stress in temporary ponds. Ecological Applications 17: 1105-1115.

ANGELER DG, M ÁLVAREZ-COBELAS, S SÁNCHEZCARRILLO (2010) Evaluating environmental conditions of a temporary pond complex using rotifer emergence from dry soils. Ecological Indicators 10: 545-549.

ARLE J (2002) Physical and chemical dynamics of temporary ponds on a calcareous plateau in Thuringia, Germany. Limnologica 32: 83-101.

ASHTON EC (2002) Mangrove sesarmid crab feeding experiments in Peninsular Malaysia. Journal of Experimental Marine Biology and Ecology 273: 97-119.

BARAN E \& J HAMBREY (1998) Mangrove conservation and coastal management in Southeast Asia: What impact on fishery resources? Marine Pollution Bulletin 37: 431-440.

BARBIER EB, M ACREMAN \& D KNOWLER (1997) Valoración económica de los humedales. Guía para decisores y planificadores. Oficina de la Convención de Ramsar. Gland, Suiza.

BERLANGA-ROBLES CA \& A RUIZ-LUNA (2004) Análisis comparativo de los sistemas clasificatorios de humedales. Instituto Nacional de Ecología, Centro de Investigación en Alimentación y Desarrollo A.C, Mazatlán, México.

BERNINGER O (1929) Wald und offenes land in Südchile seit der spanischen eroberung. Geographische Abhandlungen Stuttgart 3: 1-130.

BERTRÁN C, L VARGAS-CHACOFF, F PEÑA-CORTÉS, S MULSOW, J TAPIA, E HAUENSTEIN, R SCHLATTER, A BRAVO (2006) Macrofauna bentónica de los humedales de tres lagos salinos en el borde costero del sur de Chile. Ciencias Marinas 32: 589-596.

BOAVIDA MJ (1999) Wetlands: Most relevant structural and functional aspects. Limnetica 17: 57-63.

BOBBINK R, B BELTMAN, JTA VERHOEVEN \& DF WHIGHAM (eds) (2006) Wetlands: Functioning, diodiversity conservation and restoration. Springer, Berlin.

BOIX D, J SALA \& R MORENO-AMICH (2001) The faunal composition of espolla pond (NE Iberian peninsula): The neglected biodiversity of temporary ponds. Wetlands 21: 577-592.

BOLUND P \& S HUNHAMMAR (1999) Ecosystem services in urban areas. Ecological Economics 29: 293-301.

BOYERO L, RG PEARSON, MO GESSNER, LA BARMUTA, V FERREIRA et al. (2011) A global experiment suggests climate warming will not accelerate litter decomposition in streams but may reduce carbon sequestration. Ecology Letters 14: 289-294.

BRATTON JH (1990) Seasonal pools: An overlooked invertebrate habitat. British Wildlife 2: 22-29.

BRINSON MM (1993) A hydrogeomorphic classification for wetlands. Wetlands Research Program Technical Report WRP-DE- 4. Final Report, U.S. Army Corps of Engineers Waterways Experiment Station, Vicksburg, MS.

BRÖNMARK C \& LA HANSSON (2002) Environmental cues in lakes and ponds: Current state and perspectives. Environmental Conservation 29: 290-306.

BROOKS RT (2009) Potential impacts of global climate change on the hydrology and ecology of ephemeral freshwater systems of the forests of the northeastern United States. Climatic Change 95: 469-483.

CARPENTER SR, NF CARACO, DL CORRELL, RW HOWARTH, AN SHARPLEY \& VH SMITH (1998) Nonpoint pollution of surface waters with phosphorus and nitrogen. Ecological Applications 8: $559-568$

CASTRO C (1987) Transformaciones geomorfológicas recientes y degradación de las dunas de Ritoque. Revista de Geografía Norte Grande (Chile) 23: 63-77.

CHALAR G (2006) Dinámica de la eutrofización a diferentes escalas temporales: Embalse Salto Grande (Argentina-Uruguay). En: Tundis JG, T Matsumura-Tundisi \& C Sidagis (eds) Eutrophication in South America: Causes, consequences and technologies for management and control: 87-101. International Institute of Ecology, Inc. São Carlos, Brasil.

CHONG VC, A SASEKUMAR, MU LEH \& R D'CRUZ (1990) The fish and prawn communities of a Malaysian coastal mangrove system, with comparisons to adjacent mud flats and inshore waters. Estuarine, Coastal and Shelf Science 31: 703-722.

CLAUSEN JL, C ORTEGA, G GLAUDE, G RELYEA, G GARAY \& O GUINEO (2006) Classification of wetlands in a Patagonian national park. Wetlands 26: 217-229.

COFRÉ H \& P MARQUET (1999) Conservation status, rarity, and geographic priorities for conservation of Chilean mammals: An assessment. Biological Conservation 88: 53-68.

COMIN FA \& WD WILLIAMS (1994) Parched continents: Our common future? En: Margalef $\mathrm{R}$ (ed) Limnology now: A paradigm of planetary problems: 473-527. Elsevier, Amsterdam.

COMISIÓN NACIONAL DEL MEDIO AMBIENTE (2002) Estrategia regional de conservación y uso sustentable de la biodiversidad. Región de La Araucanía, CONAMA, Región de La Araucanía. Chile.

COMISIÓN NACIONAL DEL MEDIO AMBIENTE (2005) Estrategia nacional para la conservación y uso racional de los humedales en Chile. CONAMA, Santiago, Chile.

CONTRERAS JP (2002) Norte de Chile: Conservación de humedales altoandinos para un desarrollo productivo sustentable. Ambiente y Desarrollo (Chile) 18: 125-131

COOPS H, M BEKLIOGLU \& TL CRISMAN (2003) The role of water-level fluctuations in shallow lake 
ecosystems-workshop conclusions. Hydrobiologia 506: 23-27.

CORPORACIÓN NACIONAL FORESTAL (2010) Sistema Nacional de Áreas Silvestres Protegidas del Estado (SNASPE). Santiago, Chile. URL: http:// www.conaf.cl/parques/index.html (accedido Enero 10, 2011).

COWARDIN LM, V CARTER, FC GOLET \& ET LAROE (1979) Classification of wetlands and deepwater habitats of the United States. U.S. Department of the Interior, Fish and Wildlife Service Office of biological Services, FWS/OBS-79/31103, Washington, DC.

CRONK JK \& MS FENNESSY (2001) Wetland plants: Biology and ecology. Lewis Publishers, Boca Raton, Florida, USA.

DAVIS J, P HORWITZ, R NORRIS, B CHESSMAN, M MCGUIRE \& B SOMMER (2006) Are river bioassessment methods using macroinvertebrates applicable to wetlands?. Hydrobiologia 572: 115128.

DAVIS JA \& RH FROEND (1999) Loss and degradation of wetlands in southwestern Australia: Underlying causes, consequences and solutions. Wetlands Ecology and Management 7: 13-23.

DEL VALLE-ARANGO JI (2003) Descomposición de la hojarasca fina en bosques pantanosos del Pacífico colombiano. Interciencia 28: 148-153.

DEMERGASSO C, G CHONG, P GALLEGUILLOS, L ESCUDERO, M MARTÍNEZ-ALONSO \& I ESTEVE (2003) Tapetes microbianos del Salar de Llamará, norte de Chile. Revista Chilena de Historia Natural 76: 485-499.

DEVITOA KJ, AR HILL \& N ROULET (1996) Groundwater-surface water interactions in headwater forested wetlands of the Canadian Shield. Journal of Hydrology 181: 127-147.

DÍAZ MC, KP SMITH \& K RUTZLER (2004) Sponge species richness and abundance as indicators of mangrove epibenthic community health. Atoll Research Bulletin 518: 1-17.

DÍAZ MF, LARRAÍN J, ZEGERS G \& C TAPIA (2008) Caracterización florística e hidrológica de turberas de la Isla Grande de Chiloé, Chile. Revista Chilena de Historia Natural 81: 455-468.

DINI J, G COWAN \& P GOODMAN (1998) South African national wetland inventory. Proposed wetland classification system for South Africa. South African Wetlands Conservation Program. Pretoria, South Africa. URL: http://www.ngo. grida.no/soesa/nsoer/resource/wetland/ inventory_classif.htm (accedido Agosto 8, 2011).

DONOSO C (1981) Tipos forestales de los bosques nativos de Chile. Documento de Trabajo $\mathrm{N}^{0} 38$. Investigación y Desarrollo Forestal, Corporación Nacional Forestal y Organización de las Naciones Unidas para la Agricultura y la Alimentación. (CONAF-PNUD-FAO). Santiago, Chile.

DUBERSTEIN JA \& WH CONNER (2009) Use of hummocks and hollows by trees in tidal freshwater forested wetlands along the Savannah River. Forest Ecology and Management 258: 16131618.

DUKE NC (1992) Mangrove floristics and biogeography. En: Robertson AI \& DM Alongi (eds) Tropical mangrove ecosystems. Coastal and Estuarine Study 41: 63-100. American Geophysical Union, Washington, DC

DURÁN T, J QUIDEL \& E HAUENSTEIN (1997) Conocimientos y vivencias de dos familias
Wenteche sobre medicina mapuche. Centro de Estudios Socio-Culturales, Universidad Católica de Temuco y Ediciones LOM. Temuco, Chile.

DUSSAILLANT A, P GALDAMESB, C LE SUN (2009) Water level fluctuations in a coastal lagoon: El Yali Ramsar wetland, Chile. Desalination 246: 202-214.

ECHEVERRÍA C, A HUBERC, F TABERLETD (2007) Estudio comparativo de los componentes del balance hídrico en un bosque nativo y una pradera en el sur de Chile. Bosque 28: 271-280.

EWEL KC (1997) Water quality improvement by wetlands. In: Daily G (ed) Nature's services: Societal dependence on natural ecosystems: 329344. Island Press, Washington DC, USA.

FAUNCE CH \& SERAFY JE (2006) Mangroves as fish habitat: 50 years of field studies. Mar. Ecological Progress Series 318: 1-18.

FELLER IC \& MATHIS WN (1997) Primary herbivory by wood-boring insects along an architectural gradient of Rhizophora mangle. Biotropica 29: 440-451.

FELLER IC (2002) The role of herbivory by wood-boring insects in mangrove ecosystems in Belize. Oikos 97: 167-176.

FENNESSY S, M GERNES, J MACK \& DH WALDROP (2001) Methods for evaluating wetland condition: using vegetation to assess environmental conditions in wetlands. EPA 822-R-01-007j. U.S. Environmental Protection Agency, Office of Water, Washington DC, USA.

FERNÁNDEZ-ALÁEZ M, C FERNÁNDEZ-ALÁEZ, F GARCIIA-CRIADO \& C TRIGAL-DOMÍNGUEZ (2004) La influencia del régimen hídrico sobre las comunidades de macrófitos de lagunas someras de la Depresión del Duero. Ecosistemas (España) 13: $52-62$.

FERRIERE F (1982) Distribución, flora y ecología de los bosques pantanosos de Mirtáceas en la Región de Los Lagos, Chile. Tesis de Licenciatura, Escuela de Ingeniería Forestal, Universidad Austral de Chile, Valdivia, Chile.

FIGUEROA R, ML SUÁREZ, A ANDREU, \& VH RUIZ \& MR VIDAL-ABARCA (2009) Caracterización ecológica de humedales de la zona semiárida en Chile Central. Gayana 73: 76-94.

FISCHER S, MC MARINANE, MS FONTANARROSA, M NIEVES \& N SCWEIGMANN (2000) Urban rain pools, seasonal dynamics and entomofauna in a park of Buenos Aires. Hydrobiologia 441: 45-53.

FRAGA I ARGUIMBAU P (2008) Vascular flora associated to Mediterranean temporary ponds on the island of Minorca. Anales del Jardín Botánico de Madrid 65: 393-414.

GAFNY S \& A GASITH (1999) Spatially and temporally sporadic appearance of macrophytes in the littoral zone of lake Kinneret, Israel: Taking advantage of a window of opportunity. Aquatic Botany 62 : 249-267.

GAJARDO R (1995) La vegetación natural de Chile. Clasificación y distribución geográfica. Editorial Universitaria, Santiago, Chile.

GONZÁLEZ A \& P VITORIANO (2005) Aves de los humedales costeros de la zona de Concepción y alrededores. En: Smith-Ramírez C, JJ Armesto \& C Valdovinos (eds) Historia, biodiversidad y ecología de los bosques costeros de Chile: 485497. Universitaria Bosque Nativo, Santiago, Chile.

GONZÁLEZ M, E HAUENSTEIN, F PEÑA-CORTÉS, M GARCÍA \& O URRUTIA (2003) Comentarios sobre bosques pantanosos, humedales 
importantes del Centro-Sur de Chile. Gestión Ambiental (Chile) 9: 3-13.

HAUENSTEIN E, A. MUÑOZ-PEDREROS, F PENAA, F ENCINA \& M GONZÁLEZ (1999) Humedales: Ecosistemas de alta biodiversidad con problemas de conservación. El Árbol Nuestro Amigo (Chile) 13: 8-12.

HAUENSTEIN E, F PEÑA-CORTÉS, C BERTRÁN, J TAPIA \& R SCHLATTER (2008) Comparación florística y estado trófico basado en plantas indicadoras de lagunas costeras de la Región de La Araucanía, Chile. Ecología Austral (Chile) 18: 43-53.

HAUENSTEIN E, M GONZÁLEZ, F PEÑA-CORTÉS \& A MUÑOZ-PEDREROS (2002) Clasificación y caracterización de la flora y vegetación de los humedales de la costa de Toltén (IX Región, Chile). Gayana Botánica 59: 87-100.

HAUENSTEIN E, M GONZÁLEZ, F PEÑA-CORTÉS \& A MUÑOZ-PEDREROS (2005) Diversidad vegetal en humedales costeros de la Región de La Araucanía. En: Smith-Ramírez C, JJ Armesto \& C Valdovinos (eds) Historia, diversidad y ecología de los bosques costeros de Chile: 197-205. Editorial Universitaria, Santiago, Chile.

HAUER FR \& GA LAMBERTI (eds) (2006) Methods in stream ecology. Second edition. Elsevier, Amsterdam.

HAUER RF \& RD SMITH (1998) The hydrogeomorphic approach to functional assessment of riparian wetlands: Evaluating impacts and mitigation on river floodplains in the USA. Freshwater Biology 40: 517-530.

HILLMANN TJ \& GP QUINN (2002) Temporal change in macroinvertebrate assemblages following experimental flooding in permanent and temporary wetlands in an Australian floodplain forest. River Research and Applications 18: 137-154.

HOLLAND RF \& SK JAIN (1981) Insular biogeography of vernal pools in the Central Valley of California. American Naturalist 117: 24-37.

HUBER A, C OYARZÚN (1990) Variaciones anuales en la precipitación, escurrimiento e intercepción en un bosque de Pinus radiata. Turrialba 40: 503-508.

HUBER A, D LÓPEZ (1993) Cambios en el balance hídrico provocados por la tala rasa de un rodal adulto de Pinus radiata (D. Don). Bosque 14: 11-18.

HUECK K (1978) Los bosques de Sudamérica, ecología, conservación e importancia económica. Sociedad Alemana de Cooperación Técnica Ltda. (GTZ), Eschbor, Alemania.

HULSMANS A, B VANSCHOENWINKEL, C PYKE, BJ RIDDOCH, L BRENDONCK (2008) Quantifying the hydroregime of a temporary pool habitat: a modeling approach for ephemeral rock pools in SE Botswana. Ecosystems 111: 89-100.

INNIS SA, NAIMAN RJ \& ELLIOTT SR (2000) Indicators and assessment methods for measuring the ecological integrity of semi-aquatic terrestrial environments. Hydrobiologia 422/423: 111-131.

IUCN (2010) Red list of threatened species. Gland, Suiza. URL: http://www.iucnredlist.org (accedido Agosto 8, 2011).

JEREZ V, D LANFRANCO \& B ANDRADE (1977) Aspectos ecológicos de los ichneumónidos del Bosque de Quintero. Anales del Museo de Historia Natural de Valparaíso (Chile) 10: 161-168.

KADLEC RH (2006) Water temperature and evapotranspiration in surface flow wetlands in hot arid climate. Ecological Engineering 26: 328-340.
KEDDY PA (2000) Wetland ecology: Principles and conservation. Cambridge University Press, UK.

KOPONEN P, P NYGREN, D SABATIER, A ROUSTEAU \& E SAUR (2004) Tree species diversity and forest structure in relation to microtopography in a tropical freshwater swamp forest in French Guiana. Plant Ecology 173: 17-32.

LAMPERT TW \& U SOMMER (1997) Limnoecology: The ecology of lakes and streams. Oxford University Press, New York, USA.

LÉPEZ P (1998) Estudio fitosociológico del Parque Oncol (Valdivia, Chile). Tesis de Licenciatura, Facultad de Ciencias Forestales, Universidad Austral de Chile, Valdivia, Chile.

LI MS \& SY LEE (1997) Mangroves of China: A brief review. Forest Ecology and Management 96: 241-259

LOCKABY BG \& MR WALLBRIDGE (1998) Biogeochemistry. En: Messina MG \& WH Conner (eds) Southern forested wetlands: 149-172. Lewis publishers, Boca Raton, Florida, USA.

LUEBERT F \& PLISCOFF P (2006) Sinopsis bioclimática y vegetacional de Chile. Editorial Universitaria, Santiago, Chile.

LUGO AE, S BROWN \& MM BRINSON (1990) Concepts in wetland ecology. En: Lugo AE, MM Brinson \& S Brown (eds) Ecosystems of the world 15: Forested wetlands: 53-85. Elsevier, Amsterdam.

MARÍN VH, A TIRONI, LE DELGADO, M CONTRERAS, F NOVOA et al. (2009) On the sudden disappearance of Egeria densa from a Ramsar wetland site of southern Chile: A climatic event trigger model. Ecological Modelling 220: 17521763.

MITSCH W \& G GOSSELINK (2000) Wetlands. 3rd Ed. John Wiley \& Sons, New York, USA.

MITSCH W \& G GOSSELINK (2007) Wetlands. Fourth edition, John Wiley \& Sons Inc., New York, USA.

MITSCH W, G GOSSELINK, C ANDERSON \& L ZHANG (2009) Wetland ecosystems. John Wiley \& Sons Inc., New York, USA.

MOREAU S \& T LE TOAN (2003) Biomass quantification of Andean wetland forages using ERS satellite SAR data for optimizing livestock management. Remote Sensing of Environment 84: 477-492.

MOREAU S, R BOSSENO, X FA GU \& F BARET (2003) Assessing the biomass dynamics of Andean bofedal and totora high-protein wetland grasses from NOAA/AVHRR. Remote Sensing of Environment 85: 516-529.

MUÑOZ-PEDREROS A (2004) Los humedales del río Cruces y la Convención de Ramsar: Un intento de protección fallido. Gestión Ambiental (Chile) 10: 11-26.

MUÑOZ-PEDREROS A \& P MÖLLER (eds) (1997) Conservación de humedales. Taller Bases para la Conservación de Humedales de Chile. CEA Ediciones/Unión Mundial para la Naturaleza (UICN), Valdivia, Chile.

MUÑOZ-PEDREROS A, C GODOY \& L OLIVARES (1993) Santuario Carlos Anwandter: proposiciones para su manejo. Comunicaciones del Museo Regional de Concepción (Chile) 7: 33-47.

NAGELKERKEN I, SJM BLABER, S BOUILLON, P GREEN, M HAYWOOD et al. (2008) The habitat function of mangroves for terrestrial and marine fauna: A review. Aquatic Botany 89: 155-185.

NAIMAN RJ \& H DÉCAMPS (1997) The ecology of interfaces - riparian zones. Annual Review of Ecology, Evolution, and Systematics 28: 621-658. 
NICOLET P, J BIGGS, G FOX, MJ HODSON, C REYNOLDS, $M$ WHITFIELD \& $P$ WILLIAMS (2004) The wetland plant and macroinvertebrate assemblages of temporary ponds in England and Wales. Biological Conservation 120: 261-278.

OBERDORFER E (1960) Pflanzensoziologische studien in Chile. Flora et Vegetatio Mundi 2: 1-208.

OJEDA FP (1998) Estado actual de la diversidad biológica en Chile. Revista Chilena de Historia Natural 71: 117- 120.

PEÑA-CORTÉS F, P GUTIÉRREZ, G REBOLLEDO, M ESCALONA, E HAUENSTEIN, C BERTRÁN, R SCHLATTER \& J TAPIA (2006) Determinación del nivel de antropización de humedales como criterio para la planificación ecológica de la cuenca del lago Budi, IX Región de La Araucanía, Chile. Revista de Geografía Norte Grande (Chile) 36: 75-91.

PEROTTI MG, MC DIÉGUEZ \& FG JARA (2005) Estado del conocimiento de humedales del norte patagónico (Argentina): Aspectos relevantes e importancia para la conservación de la biodiversidad regional. Revista Chilena de Historia Natural 78: 723-737.

PISANO E (1954) La vegetación de las distintas zonas geográficas chilenas. Revista Geográfica de Chile 11: $95-106$

RAMÍREZ C \& C SAN MARTÍN (2005) Asociaciones vegetales de la cordillera de la Costa de la Región de Los Lagos. En: Smith-Ramírez C, J Armesto \& C Valdovinos (eds) Historia diversidad y ecología de los bosques costeros de Chile: 206-223. Editorial Universitaria, Santiago, Chile.

RAMÍREZ C (1982) Pasado, presente y futuro de la vegetación nativa del sur de Chile. Creces (Chile) 3: $40-45$.

RAMÍREZ C, C SAN MARTÍN \& H RUBILAR (2002) Una propuesta para la clasificación de los humedales chilenos. Revista Geográfica de Valparaíso (Chile) 33: 265-273.

RAMÍREZ C, C SAN MARTÍN \& J SAN MARTÍN (1995) Estructura florística de los bosques pantanosos de Chile sur-central. En: Armesto J, C Villagrán \& MK Arroyo (eds) Ecología de los bosques nativos de Chile: 215-234. Editorial Universitaria, Santiago, Chile.

RAMÍREZ C, F FERRIERE \& H FIGUEROA (1983) Estudio fitosociológico de los bosques pantanosos templados del sur de Chile. Revista Chilena de Historia Natural 56: 11-26.

RAMÍREZ C (1988) Toponimia indígena de Chile. Nombres de lugares indígenas de Osorno, Llanquihue y Chiloé (Chile). Universidad Austral de Chile, Dirección de Investigación, UNIPRINT, Chile.

RAMSAR (1996) Plan Estratégico 1997-2002. Oficina de la Convención de Ramsar, Gland, Suiza.

RAMSAR (2006) Manual de la Convención de Ramsar: Guía a la convención sobre los humedales (Ramsar, Irán, 1971), cuarta edición. Secretaría de la Convención de Ramsar, Gland, Suiza.

REDDY R \& R DELAUNE (2008) Biogeochemistry of wetlands. Science and applications. Taylor \& Francis Group, Boca Raton, New York, London.

REICHE K (1907) Grundzüge der pflanzenverbreitung in Chile. Vegetation der Erde, Leipzig 8: 1-374.

REISS KC (2006) Florida wetland condition index for depressional forested wetlands. Ecological Indicators 6: 337-352.
ROULET NT (1991) Stormflow production in a headwater basin swamp. Nordic Hydrology 22: 161-174.

SALA OE, FS CHAPIN, JJ ARMESTO, E BERLOW, J BLOOMFIELD et al. (2000) Global biodiversity scenarios for the year 2100. Science 287: 17701774.

SAN MARTÍN C, C RAMÍREZ, D CONTRERAS (2006) Una nueva asociación boscosa pantanosa para Chile Caldcluvio-Lumetum gayanae. Revista Geográfica de Valparaíso (Chile) 37: 77-87.

SAN MARTÍN J, A TRONCOSO \& C RAMÍREZ (1988) Estudio fitosociológico de los bosques pantanosos nativos de la cordillera de la Costa en Chile central. Bosque 9: 17-33.

SAN MARTÍN J, A TRONCOSO, C RAMÍREZ, C SAN MARTÍN \& A DUARTE (1990) Estudio florístico $\mathrm{y}$ vegetacional de los bosques pantanosos nativos de la cordillera costera entre los ríos Rapel y Mataquito, Chile central. Revista Geográfica de Chile, Terra Australis 33: 103-128.

SAN MARTÍN C, D CONTRERAS, J SAN MARÍN \& C RAMÍREZ (1992a) Vegetación de las marismas del centro-sur de Chile. Revista Chilena de Historia Natural 65: 327-342.

SAN MARTÍN J, J SOLERVICENS, C RAMÍREZ, C SAN MARTIN \& M ELGUETA (1992b) Estudio fitosociológico de los bosques pantanosos de mirtáceas de la Región del Maule, Chile. Ciencias Forestales 8: 3-18.

SEREY I \& R VILLASEÑOR (1977) La vegetación boscosa de la costa y el valle central de la provincia de Llanquihue (X Región). Anales del Museo de Historia Natural de Valparaíso (Chile) 10: $39-44$

SERRANO L \& L SERRANO (1995) Influences of groundwater exploitation for urban water supply on temporary ponds from the Donana National Park (Southwest Spain). Journal of Environmental Management 46: 229-238.

SIEGEL DI (1988) The recharge-discharge function of wetlands near Juneau, Alaska: Part 1. Hydrogeological investigations. Ground Water 26: 427-434.

SOLERVICENS J \& M ELGUETA (1994) Insectos de follaje de bosques pantanosos del Norte Chico, centro y sur de Chile. Revista Chilena de Entomología (Chile) 21: 135-164.

SOLERVICENS J (1973) Coleópteros del bosque de Quintero. Anales del Museo de Historia Natural de Valparaíso (Chile) 6: 115-159.

TAVERNINI S (2008) Seasonal and inter-annual zooplankton dynamics in temporary pools with different hydroperiods. Limnologica 38: 63-75.

TAVERNINI S, G MURA \& G ROSSETTI (2005) Factors influencing the seasonal phenology and composition of zooplankton communities in mountain temporary pools. International Review of Hydrobiology 90: 358-375.

TEILLIER S \& P BECERRA (2003) Flora y vegetación del salar de Ascotán, Andes del norte de Chile. Gayana Botánica 60: 114-122.

TEILLIER S (1998) Flora y vegetación altoandina del área de Collahuasi - Salar de Coposa, Andes del norte de Chile. Revista Chilena de Historia Natural 71: 313-329.

TENEB E, P GÓMEZ \& M GONZÁLEZ (2008) Observaciones sobre la flora y vegetación de dos turberas en la Región de Aysén, patagonia chilena. Gayana Botánica 65: 229-232. 
THONG KL \& A SASEKUMAR (1984) The trophic relationships of the fish of the Angsa Bank, Selangor, Malaysia. En: Soepadmo A, AN Rao \& DJ MacIntosh (eds) Proceedings of the Asian Symposium on the mangrove environmental. Research and management: 385-399. University of Malaya and UNESCO, Kuala Lumpur, Malaysia.

TUNDISI JG \& M MATSUMURA-TUNDISI (1990) Limnology and eutrophication of Barra Bonita Reservoir, Sao Paulo State, Southern Brazil, Archiv für Hydrobiologie Beihefte Ergebnisse der Limnologie 33: 661-676.

URRUTIA J (2008) Flora, fitosociología y estado de conservación de rodales representativos de bosque pantanoso de la depresión central, provincia de Cautín, Región de La Araucanía, Chile. Tesis de Licenciatura, Escuela de Ciencias Ambientales, Facultad de Recursos Naturales, Universidad Católica de Temuco.

VARELA J (1981) Geología del cuaternario del área de los Vilos - Ensenada el Negro (IV Región) y su relación con la existencia del bosque "relicto" de Quebrada Quereo. Congreso Internacional de Zonas Áridas y Semiáridas. La Serena, Chile. Comunicaciones (Chile) 33: 17-30.

VILLAGRA J, D MONTENEGRO, C SAN MARTÍN, C RAMIREZ \& I ÁLVAREZ (2009) Estudio de la flora liquénica de las turberas de la comuna de Tortel (Región de Aysén), Patagonia Chilena. Anales Instituto Patagonia (Chile) 37: 53-62.

VILLAGRÁN C (1982) Estructura florística e historia del bosque pantanoso de Quintero (Chile, V Región) y su relación con las comunidades relictuales de Chile central y Norte Chico. Actas III Congreso Geológico Chileno 3: 377-402.

VILLAGRÁN-MELLA R, AGUAYO M, PARRA L \& GONZÁLEZ A (2006) Relación entre características del hábitat y estructura del ensamble de insectos en humedales palustres urbanos del centro-sur de Chile. Revista Chilena de Historia Natural 79: 195-211.

VILLAGRÁN-MELLA R, ME CASANUEVA \& LE PARRA (2005) Mites in the parenchyma of Juncus procerus in marshey wetlands in the Bio Bio region, Chile. Gayana 69: 22-26.

VILLA-MARTÍNEZ R \& C VILLAGRÁN (1997) Historia de la vegetación de los bosques pantanosos de la costa de Chile central durante el Holoceno medio y tardío. Revista Chilena de Historia Natural 70: 391-401.

VIVIAN-SMITH G (1997) Microtopographic heterogeneity and floristic diversity in experimental wetland communities. Journal of Ecology 85: 71-82.

WARNER BG \& CDA RUBEC (1997) The Canadian Wetland Classification System. Second Edition. National Wetlands Working Group, Wetlands Research Centre, University of Waterloo, Waterloo, Ontario, Canada.

WIGGINS B, RJ MACKAY \& IM SMITH (1980) Evolutionary and ecological strategies of animals in annual temporary pools. Archiv für Hydrobiologie Supplement 125: 85-110.

ZAPATA J, M YÁÑEZ \& E RUDOLPH (2008) Tecamebianos (Protozoa: Rhizopoda) de una turbera del parque nacional Puyehue $\left(40^{\circ} 45^{\prime} \mathrm{S}\right.$; $72^{\circ} 19^{\prime}$ W), Chile. Gayana 72: 9-17.

ZEDLER PH (1987) The ecology of southern California vernal pools: A community profile. Biological Report 85 (7.11.). Prepared for USFWS, National Wetlands Research Center, Washington DC.

ZEGERS G, J LARRAÍN, F DÍAZ \& J ARMESTO (2006) Impacto ecológico y social de la explotación de pomponales y turberas de Sphagnum en la isla Grande de Chiloé. Ambiente y Desarrollo (Chile) 22: 28-34. 